\title{
Validation of a Mouse Adhesion Reduction Model Using Seprafilm ${ }^{\circledR}$
}

\author{
ABRAHAM N. MORSE, M.D., ${ }^{1}$ ROBERT A. HAMMER, M.D., ${ }^{2}$ \\ JEFFREY L. CORNELLA, M.D., ${ }^{3}$ and JOSEPH C. LOFTUS, Ph.D. ${ }^{4}$
}

\begin{abstract}
Introduction: Most initial trials of antiadhesion technologies are currently carried out in rats or rabbits. This paper describes a simple, reliable technique for producing intraperitoneal adhesions in the mouse. This model has been validated using a hyaluronan/carboxymethylcellulose (HA/CMC) barrier (Seprafilm ${ }^{\circledR}$; Genzyme, Somerville, NJ) currently in clinical use. Methods: Adult, FVB mice were anesthetized with isoflurane. Celiotomy was performed in each mouse and the cecum and abdominal wall were abraded with sandpaper in a standardized manner. The mice either received no treatment or the cecum was wrapped in the HA/CMC membrane. Treatment groups were assigned, after abrasion, by coin toss. One (1) week later, the mice were euthanized and the adhesions were scored by two trained investigators blinded to the treatment group of each animal and the other investigator's adhesion score. Results: Maximum adhesion grades were significantly lower $(p=0.009$, Wilcoxon rank-sum) for the HA/CMC group (median $=0 ; \mathrm{s}=1.21 ; n=18$ ) than for the control group ( mean $=2 ; \mathrm{s}=0.85 ; n=24$ ). The risk of any adhesion formation for the HA/CMC group was only half (relative risk $[R R]=0.51,95 \%$ confidence interval $[C I]=0.30-0.87$ ) as large as the risk of adhesion formation in the control group. Eight (8) mice (16\%) died during the experiment. The risk of death was 8 times higher for mice treated with HA/CMC membrane than for the controls $(\mathrm{RR}=7.7 ; 95 \% \mathrm{CI}=1.0-57)$, suggesting that treatment with HA/CMC could have increased the risk of mortality. Conclusions: This simple technique for adhesion formation is reliable and allows fast throughput of animals. The extent of adhesion prevention with HA/CMC membrane suggests that trials of adhesion prevention technologies in this model system may mirror those seen in humans. (J GYNECOL SURG 21:147)
\end{abstract}

\section{INTRODUCTION}

\footnotetext{
A BDOMINAL ADHESIONS are the leading cause of bowel obstruction in the developed world. ${ }^{1} \mathrm{~A}$ recent survey estimated that there are 12-14,000 cases of adhesive bowel obstruction annually in the United Kingdom. ${ }^{2}$ In the United States, there were more than 130,000 hospital admis-

${ }^{1}$ Department of Obstetrics and Gynecology, University of Massachusetts Medical Center / UMass Memorial Medical Center, Worcester, MA.

${ }^{2}$ Department of Obstetrics and Gynecology, Northwestern University Medical Center, Chicago, IL.

${ }^{3}$ Division of Pelvic Reconstructive Surgery, Mayo Clinic Scottsdale, Scottsdale, AZ.

${ }^{4}$ Department of Biochemistry and Molecular Biology, Mayo Clinic Scottsdale, Scottsdale, AZ.
} 
sions for adhesive bowel obstruction in 1990. One percent (1\%) of all surgical admissions and 3 percent of all laparotomies were secondary to complications from adhesive disease. In the United States, in 1988, the direct hospital costs for the surgical treatment of abdominal adhesions exceeded $\$ 1.1$ billion. $^{3}$

Surgeons and surgical researchers have worked to understand the etiology of adhesions and prevent their formation throughout the modern era of surgery. In the past decade, significant progress has been made with the development of adhesion barriers. These include Gore-Tex ${ }^{\circledR}$ membrane; oxidized, regenerated cellulose (Interceed ${ }^{\circledR}$ ); and hyaluronic acid/carboxymethylcellulose membrane (Seprafilm ${ }^{\circledR}$; Genzyme, Somerville, NJ). Hyaluronic acid (HA) has been shown to prevent adhesion formation in both experimental models and human subjects. ${ }^{4-7}$

Following the introduction of these antiadhesion products, there has been a significant increase in the research and development of antiadhesion technologies. Each new substance or drug must be tested in animals before consideration of clinical trials can begin. To date, most small-animal models reported in the literature have involved rabbits and rats. Several papers have reported the ability to generate consistent levels of intra-abdominal or uterine adhesions in mice. ${ }^{8-11} \mathrm{~A}$ reliable mouse model would allow faster and less-expensive evaluation of adhesion-reduction technologies. However, to be useful in evaluating the clinical potential of a new substance or device, any animal model should ideally be shown to respond to established adhesion production methods and antiadhesion interventions in a manner similar to humans, otherwise the applicability of the conclusions regarding the utility of intervention in that model may be spurious. ${ }^{12}$ However, none of the mouse models have been validated using a substance with demonstrated adhesion prevention properties in humans. This paper discusses a simple, fast, and reliable technique for abdominal adhesion development in the mouse which we have validated using a hyaluronan/carboxymethylcellulose barrier (HA/CMC) currently in clinical use.

\section{METHODS}

Adult, male and female FVB mice were used in these studies. All animal protocols were approved by the Mayo Clinic Institutional Animal Care and Use Committee. Surgeries were carried out in a laminar flow hood with sterile gloves, masks, and gowns. Instruments were bead sterilized at $150^{\circ} \mathrm{C}$ between animals.

Anesthesia was administered by placing the animals in a gas-tight Plexiglas chamber with circulating 2\%-3\% isoflurane. Animals were generally unconscious within 60 seconds, at which point, they were removed from the chamber and placed on the operating surface with a face cone (Figure 1) providing 2\%-3\% isoflurane for the duration of the procedure. After an initial learning curve, the induction, celiotomy, abrasion, and closure of each animal took 6-7 minutes. With one assistant helping the surgeon by performing the induction, depilation, and abdominal preparation, an average of 8-9 mice were operated on in 1 hour. Animals generally awoke and were ambulating and drinking within 5 minutes of the cessation of anesthesia.

Once adequate anesthesia was established, as judged by respiratory rate and depth, the skin was chemically depilated (Nair ${ }^{\circledR}$, Carter-Wallace, Inc., Cranbury, NJ), cleansed with Povidone/iodine, and swabbed with $70 \%$ ethyl alcohol ETOH. Each mouse was draped with a small fenestrated sterile sheet and a 1-2-cm incision was made in the left upper quadrant (Fig. 1). A $1 \times 1 \mathrm{~cm}$ sheet of autoclaved 400-grit aluminum oxide sandpaper was then used to abrade the anterior abdominal wall overlying the intra-abdominal location of the cecum by passing it across the site once with gentle pressure. This was repeated 5 additional times. The cecum was then exteriorized onto the sterile sheet and the same sandpaper was used to abrade the anterior and posterior surfaces of the cecum by passing the sandpaper across each surface 6 times (Fig. 2). This consistently de- 


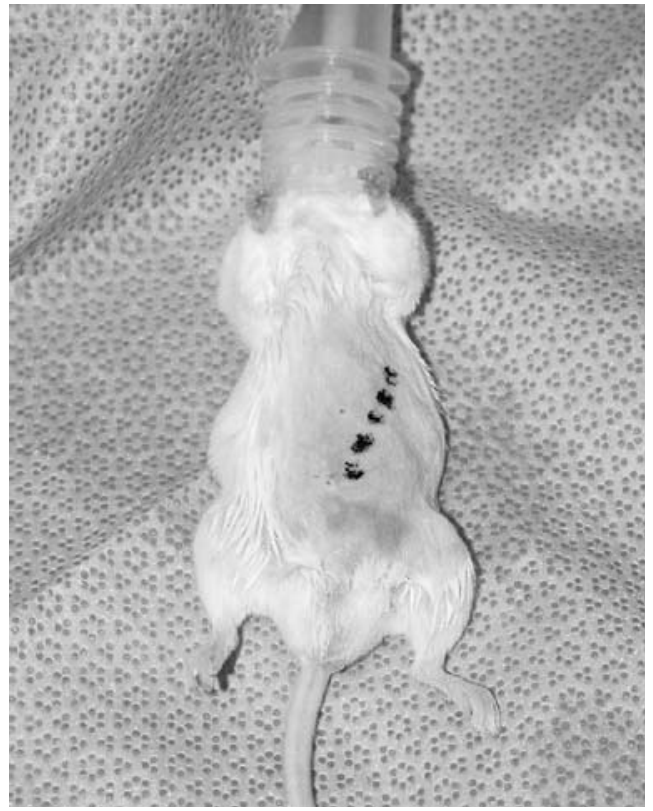

FIG. 1. Anesthetized mouse with face-cone for isoflurane anesthesia in place. Incision for adhesion creation is marked on the abdomen (dotted line).

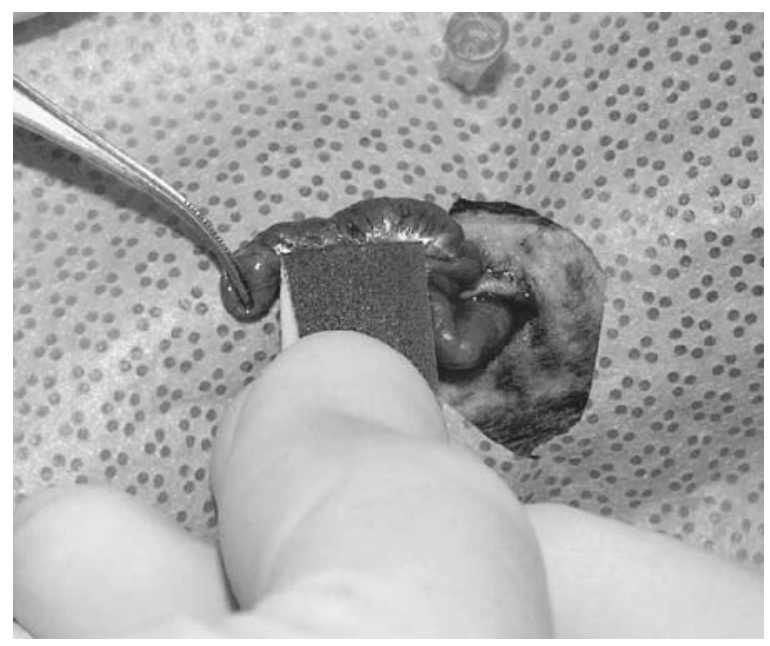

FIG. 2. Adhesion creation technique using sterile sandpaper to deserosalize the cecum.

serosalized the cecum and created punctate bleeding. The group assignments were made as follows: After the incision and abrasion had been completed, each animal was randomized by coin flip to receive HA/CMC (HA/CMC group) or nothing (control group). At the time of sacrifice and scoring, the investigators were blinded to each animal's treatment group assignment.

If $\mathrm{HA} / \mathrm{CMC}$ membrane was applied, a $2 \times 2 \mathrm{~cm}$ sterile piece was wrapped around the cecum and moistened with 3 drops of saline prior to its replacement into the peritoneal cavity. Control animals received 3 drops of saline into the peritoneal cavity prior to closure. The incision was closed using a running, 4-0 polyglycolic acid for the fascia and skin. Animals received buprenorphine $0.25 \mathrm{mg} / \mathrm{kg}$ immediately prior to incision and acetaminophen $1 \mathrm{mg} / \mathrm{mL}$ in their drinking water for the first 3 postoperative days. On postoperative day number 7, the mice were euthanized using $5 \%$ carbon dioxide gas and necropsy was performed.

We used a 4-grade adhesion scoring system for grading the severity of adhesions involving the cecum based on that found by Montz et al. ${ }^{8}$ At necropsy, 2 trained observers independently recorded their adhesion scores without discussion. Differences were resolved by assigning the mean adhesion score to that mouse. No adhesion scores for the same animal differed by more than 1 grade. The scoring system was defined as follows:

Grade 0-no adhesions

Grade 1-filmy adhesions, easily separated with gentle traction

Grade 2-moderate adhesions, requiring significant traction to separate

Grade 3-dense adhesions, unable to separate without tearing underlying involved structures.

\section{Statistical analysis}

Our initial pilot study of 16 animals suggested that 22 mice were needed in each group to have $80 \%$ power to detect a 1-grade reduction in adhesion formation. The difference in adhesion grade 
between the control and HA/CMC groups was compared using the Wilcoxon rank-sum test. The Fisher's exact test was used to compare the incidence of 0 adhesions in the two groups. The $\kappa$ statistic was used to assess interobserver reliability of the adhesion scoring system.

\section{RESULTS}

\section{Adhesion formation technique}

Initial experiments suggested that cecal abrasion alone was insufficient for reliable adhesion formation. Several different protocols were assessed for their ability to reliably create grade- 2 or greater adhesions without causing a high percentage of intraoperative bowel perforation. Figure 3 shows the median adhesion score for 4 different protocols that were investigated. Based on these data, the technique of cecal abrasion using 400-grit sandpaper plus anterior abdominal-wall abrasion was chosen for all subsequent investigations. This protocol reliably created grade $2-3$ adhesions without causing bowel perforation or cecal hemorrhage as in the appendiceal and 320 grit sandpaper groups respectively.

\section{Adhesion prevention by $H A / C M C$ membrane}

Maximum adhesion grades were significantly lower ( $p=0.009$, Wilcoxon rank-sum) for the $\mathrm{HA} / \mathrm{CMC}$ group $($ median $=0 ; \mathrm{s}=1.21 ; n=18$ ) than for the control group (median $=2 ; \mathrm{s}=$ $0.85 ; n=24)$. The mean difference between groups was 1.0 grades (95\% confidence interval $[C I]=0.4-1.6$ grades). The distributions of adhesion grades are shown in Figure 4. The HA/CMC treated group had a significantly higher ( $p=0.006$; Fisher's exact test) percentage of mice with no adhesions $(56 \%, 10 / 18)$ than the control group $(12 \% ; 3 / 24)$. The risk of any adhesion forma-

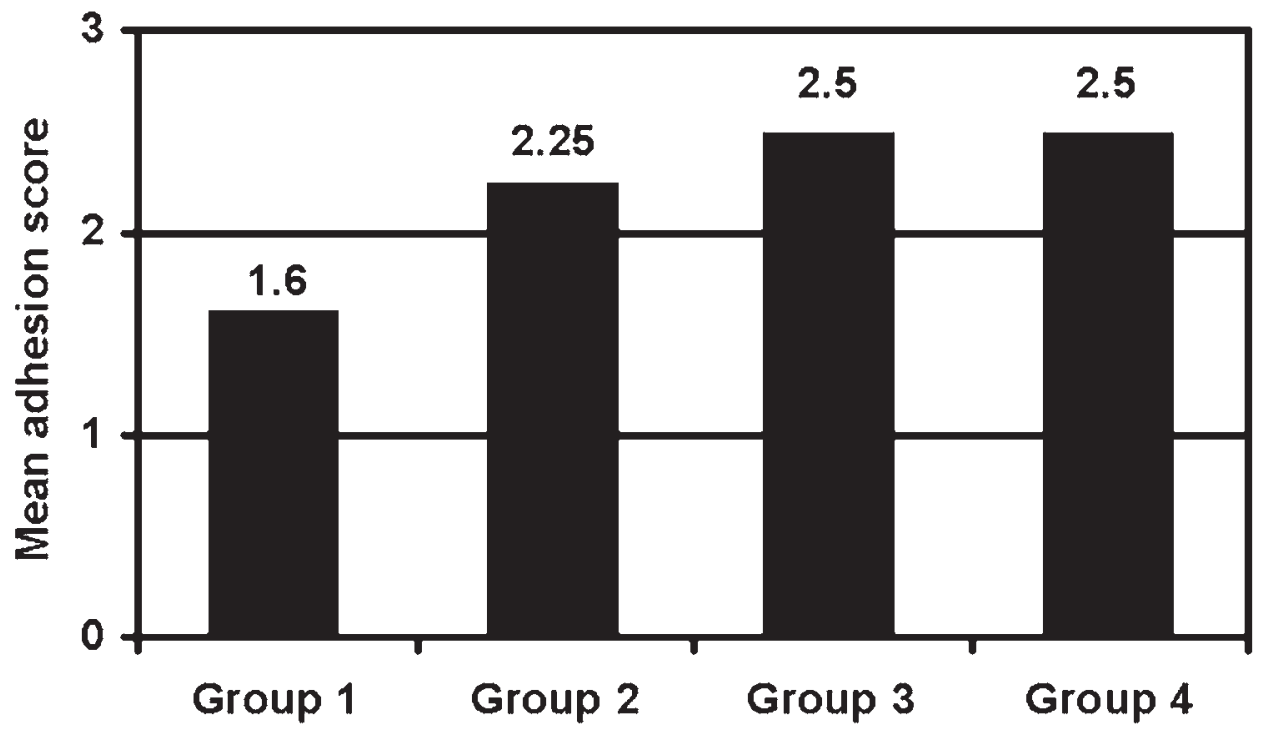

FIG. 3. Comparison of adhesion creation techniques. Group 1: 400-grit sandpaper, cecal abrasion only. Group 2: 400grit sandpaper, abrasion to cecum and anterior abdominal wall (AAW). Group 3: 320-grit and AAW abrasion. Group 4: 400 grit + peritoneal excision. 


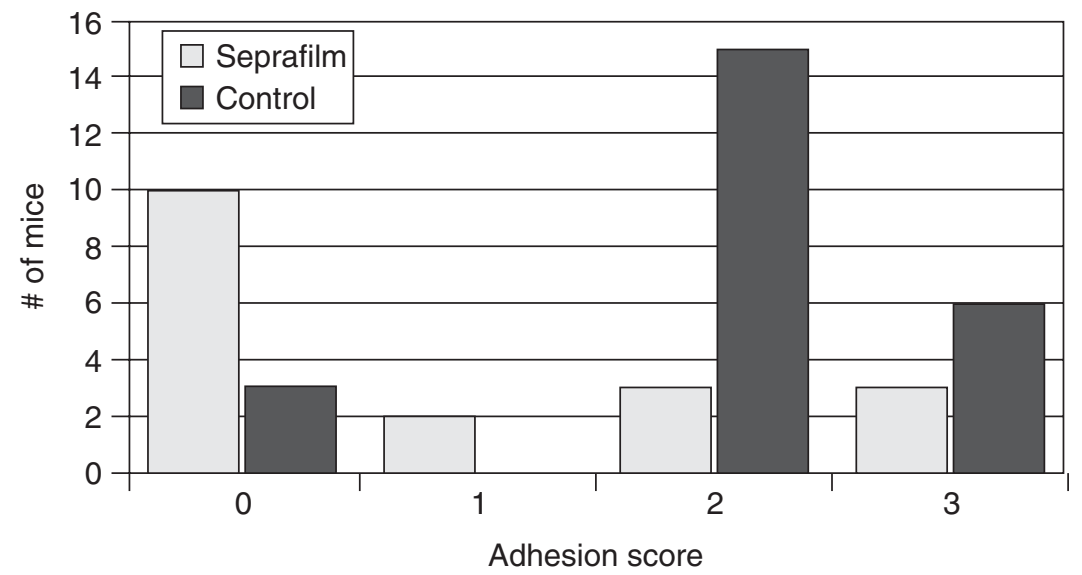

FIG. 4. Adhesion scores for hyaluronan/carboxymethylcellulose (HA/CMC; barrier (Seprafilm ${ }^{\circledR}$, Genzyme, Somerville, NJ) animals (light gray) versus controls (black). Maximum adhesion grades were significantly lower $(p=$ $0.009)$ for the HA/CMC group. The mean difference between groups was 1.0 grades $(95 \%$ confidence interval $=0.4-1.6$ grades).

tion for the HA/CMC treated group $(44 \% ; 8 / 18)$ was only half (relative risk $[\mathrm{RR}]=0.51 ; 95 \%$ $\mathrm{CI}=0.30-0.87)$ as large as the risk of adhesion formation in the control group $(88 \% ; 21 / 24)$.

\section{Morbidity and mortality}

A total of 8 mice $(16 \%)$ died during the experiment. A significantly higher proportion ( $p=$ 0.024 , Fisher's exact test) of mice died in the HA/CMC group $(31 \% ; 8 / 26)$ than in the control group $(0 \% ; 0 / 25)$. The risk of death was 8 times higher for mice treated with HA/CMC membrane than for the controls $(\mathrm{RR}=7.7 ; 95 \%$; $\mathrm{CI}=1.0$ to 57 ), suggesting that treatment with $\mathrm{HA} / \mathrm{CMC}$ increased the risk of mortality. Seven (7) mice that died during the experiment had grade- 1 or grade- 0 adhesions at autopsy. One mouse that died had grade- 3 adhesions and a pericecal abscess.

\section{Interobserver reliability}

Weighted $\kappa$ coefficient for interobserver reliability was 0.962 (95\% CI; 0.947-0.977).

\section{DISCUSSION}

In this article we have presented a validated method for assessing adhesion-prevention interventions in a mouse cecal-abrasion model. Our findings of a $50 \%$ reduction in adhesion formation with HA/CMC membrane are similar to those reported in several of the randomized, clinical trials with Seprafilm, ${ }^{6,13}$ suggesting that this model may provide reasonable sensitivity and specificity for evaluating new adhesion-prevention interventions. The technique utilizes simple tools and anesthetic and surgical techniques that allow for fast throughput of animals. The scoring technique is easily learned and reliable. A posthoc power analysis indicates that 2 groups of 20 mice 
should be sufficient for most evaluations. We purposely did not create a highly quantitative system of abrasion in order to keep the protocol simple. The abrasion protocol could be modified to include a measured applied pressure and/or a standardized area on the anterior abdominal wall and cecum for abrasion. Nonetheless, the amount of abrasion produced did appear to be reproducible.

The markedly increased mortality in the HA/CMC arm of the study is a concern for two reasons. First, it may have biased our results if mice with a higher adhesion grade were more likely to die, thus, artificially lowering the apparent adhesion score in the HA/CMC group. Second it raises questions as to whether the increased mortality might represent a real effect of HA/CMC application and thus have negative implications for its clinical use. Review of the literature does identify several clinical case reports and experimental series that also demonstrated an association between HA/CMC application and an increased incidence of inflammation or intraperitoneal infection. ${ }^{14-17}$ However, the mortality in the HA/CMC group was concentrated in the first several groups of animals that we tested. Death rates in subsequent experiments with Seprafilm using the same technique have been negligible. Thus, it may be that, as our technique improved with practice, no real additional mortality risk was associated with HA/CMC application.

Current models of adhesion formation and prevention are based on a standardized intervention that creates intra-abdominal adhesions and assesses the ability of a technology to reduce the extent and tenacity of adhesion formation. It is important to remember that adhesion formation per se is both ubiquitous after celiotomy and is most often without clinical consequences. The major source of morbidity and mortality from adhesion formation results from intestinal obstruction, but none of the current models (including the one discussed in this article) and none of the published clinical trials has attempted to assess the impact of adhesion-prevention technology on the development of intestinal obstruction. The commercially available products were approved based on their ability to reduce adhesion formation. The mouse model presented in this article should be helpful for accelerating and lowering the cost of investigating new adhesion-prevention technologies by providing a simple, fast throughput technique, using mice, that appears to be predictive of behavior in human models. However, future development of adhesion models should also focus on creation of an adhesive-obstruction model. A reliable model of adhesive intestinal obstruction would be invaluable for identifying interventions that truly prevent the most serious complication of adhesion formation.

\section{REFERENCES}

1. Ellis H. The causes and prevention of intestinal adhesions. Br J Surg 1982;69:241.

2. Holmdahl L, Risberg B, Beck DE, et al. Adhesions: Pathogenesis and prevention-panel discussion and summary. Eur J Surg 1997;577(Suppl):56.

3. Ray NF, Larsen JW, Stillman RJ, Jacobs RJ. Economic impact of hospitalizations for lower abdominal adhesiolysis in the United States in 1988. Surg Gynecol Obstet 1993;176:271.

4. Diamond MP. Seprafilm ${ }^{\circledR}$ Adhesion Study Group. Seprafilm ${ }^{\text {TM }}$ (HAL-F) reduces postoperative adhesions: Initial results of a multicenter gynecologic clinical study. 4th Congress of the European Society for Gynaecological Endoscopy, Brussels, Belgium, December 6-9, 1995: Abstract O53.

5. Goldberg EP, Burns JW, Yaacobi Y. Prevention of postoperative adhesions by precoating tissues with dilute sodium hyaluronate solutions. In: Diamond MP, ed. Gynecologic Surgery and Adhesion Prevention: Proceedings of the Second International Symposium on Gynecologic Surgery and Adhesion Prevention. Palm Beach, FL, January 1992; Philadelphia:Wiley-Liss, 1993:191.

6. Becker JM, Dayton MT, Fazio VW, et al. Prevention of postoperative abdominal adhesions by a sodium hyaluronatebased bioresorbable membrane: A prospective randomized, double-blind multicenter study. J Am Coll Surg 1996;183:297. 
7. Vrijland WW, Tseng LN, Eijkman HJ, et al. Fewer intraperitoneal adhesions with use of hyaluronic acid-carboxymethylcellulose membrane: A randomized clinical trial. Ann Surg 2002;235:193.

8. Montz FJ, Gotlieb WH, Holschneider $\mathrm{CH}$, et al. Interleukin 10: Ability to minimize postoperative intraperitoneal adhesion formation in a murine model. Fertil Steril 1999;61:1136.

9. Haney AF, Doty E. A barrier composed of chemically cross-linked hyaluronic acid (Incert) reduces postoperative adhesion formation. Fertil Steril 1998;70:145.

10. Saltzman AK, Olson TA, Mohanraj D, et al. Prevention of postoperative adhesions by an antibody to vascular permeability factor/vascular endothelial growth factor in a murine model. Am J Obstet Gynecol 1996;174:1502.

11. Sawada T, Hasegawa K, Tsukada K, Kawakami S. Adhesion preventive effect of hyaluronic acid after intraperitoneal surgery in mice. Hum Reprod 1999;14:1470.

12. Wiseman DM. Correlations between animal and human models of adhesions. Proceedings of the Fifth International Congress on Peritoneal Repair and Adhesions (PAX), 1999.

13. Diamond MP, and the Sepracoat Adhesion Study Group. Reduction of de novo postsurgical adhesions by intraoperative precoating with Sepracoat ${ }^{\circledR}$ (HAL-C) solution: A prospective, randomized, blinded, placebo-controlled multicenter study. Fertil Steril 1998;69:1067.

14. Klingler PJ, Floch NR, Seelig MH, et al. Seprafilm ${ }^{\circledR}$-induced peritoneal inflammation: A previously unknown complication. Dis Colon Rectum 1999;42:1639.

15. Tzianabos AO, Cisneros RL, Gershkovich J, et al. Effect of surgical adhesion reduction devices on the propagation of experimental intra-abdominal infection. Arch Surg 1999;134:1254.

16. Bowers D, Raybon RB, Wheeless CR. Hyaluronic acid-carboxymethylcellulose film and perianastomotic adhesions in previously irradiated rats. Am J Obstet Gynecol 1999;181:1335.

17. Moreira H, Wexner SD, Yamaguchi $\mathrm{T}$, et al. Use of bioresorbable membrane (sodium hyaluronate + carboxymethylcellulose) after controlled bowel injuries in a rabbit model. Dis Colon Rectum 2000;43:182.

Address reprint requests to:

Abraham N. Morse, M.D.

Department of Obstetrics and Gynecology

University of Massachusetts Medical Center

UMass Memorial Medical Center

Jaquith 435

119 Belmont Street

Worcester, MA 01605

E-mail:morsea@ummhc.org 